\title{
Randomised controlled trial of non-invasive ventilation (NIV) for nocturnal hypoventilation in neuromuscular and chest wall disease patients with daytime normocapnia
}

\author{
S Ward, M Chatwin, S Heather, A K Simonds
}

Thorax 2005;60:1019-1024. doi: 10.1136/thx.2004.037424

See end of article for
authors' affiliations
.....................
Correspondence to:
Dr A K Simonds, Clinical
and Academic Department
of Sleep and Breathing,
Royal Brompton \&
Harefield NHS Trust,
Sydney Street, London
SW3 6NP, UK; A.
Simonds@rbht.nhs.uk
Received 9 November
2004
Accepted 9 May 2005

\begin{abstract}
Background: Long term non-invasive ventilation (NIV) reduces morbidity and mortality in patients with neuromuscular and chest wall disease with hypercapnic ventilatory failure, but preventive use has not produced benefit in normocapnic patients with Duchenne muscular dystrophy. Individuals with nocturnal hypercapnia but daytime normocapnia were randomised to a control group or nocturnal NIV to examine whether nocturnal hypoventilation is a valid indication for NIV.

Methods: Forty eight patients with congenital neuromuscular or chest wall disease aged 7-51 years and vital capacity $<50 \%$ predicted underwent overnight respiratory monitoring. Twenty six with daytime normocapnia and nocturnal hypercapnia were randomised to either nocturnal NIV or to a control group without ventilatory support. NIV was started in the control group if patients fulfilled preset safety criteria. Results: Peak nocturnal transcutaneous carbon dioxide tension $\left(\mathrm{TcCO}_{2}\right)$ did not differ between the groups, but the mean (SD) percentage of the night during which $\mathrm{TcCO}_{2}$ was $>6.5 \mathrm{kPa}$ decreased in the NIV group $(-57.7(26.1) \%)$ but not in controls $(-11.75(46.1) \% ; p=0.049,95 \% \mathrm{Cl}-91.5$ to -0.35$)$. Mean (SD) arterial oxygen saturation increased in the NIV group (+2.97 (2.57)\%) but not in controls (-1.12 (2.02)\%; $\mathrm{p}=0.024,95 \% \mathrm{Cl} 0.69$ to 7.5$)$. Nine of the 10 controls failed non-intervention by fulfilling criteria to initiate NIV after a mean (SD) of 8.3 (7.3) months.

Conclusion: Patients with neuromuscular disease with nocturnal hypoventilation are likely to deteriorate with the development of daytime hypercapnia and/or progressive symptoms within 2 years and may benefit from the introduction of nocturnal NIV before daytime hypercapnia ensues.
\end{abstract}

$\mathrm{R}$ espiratory muscle weakness is inevitable in many congenital neuromuscular and chest wall disorders and is the most common cause of chest infections, hospital admissions, and premature death. Treatment of chronic ventilatory failure with nocturnal non-invasive ventilation (NIV) in adults and children with hypercapnic respiratory failure secondary to neuromuscular and chest wall disorders is standard practice. Application of nocturnal NIV when these individuals become hypercapnic during the day prolongs survival, $^{12}$ improves nocturnal and diurnal blood gas tensions, nomalises sleep patterns, ${ }^{3}$ and enhances health related quality of life. ${ }^{2}$ Conversely, preventive use of NIV in patients with asymptomatic Duchenne muscular dystrophy before the development of nocturnal or diurnal ventilatory insufficiency in one randomised trial $^{4}$ did not improve survival or prevent deterioration in pulmonary function, and was poorly tolerated. An American Thoracic Society Consensus statement ${ }^{5}$ recommends starting NIV when daytime hypercapnia is present; however, some patients experience uncontrolled respiratory decompensation or are markedly symptomatic from nocturnal hypoventilation before the development of diurnal ventilatory failure. In addition, further Consensus Conference guidelines ${ }^{6}$ suggest starting NIV in progressive conditions when the maximum inspiratory pressure is less than $60 \mathrm{~cm} \mathrm{H}_{2} \mathrm{O}$, forced vital capacity is less than $50 \%$ predicted, or if nocturnal arterial oxygen saturation is less than $88 \%$ for more than five consecutive minutes. None of these recommendations is based on controlled studies so the evidence to support the use of NIV to correct sleep disordered breathing in neuromuscular and chest wall disorders is limited. A Cochrane review ${ }^{7}$ has concluded that randomised trials are needed to confirm the benefit of mechanical ventilation in patients with chronic hypoventilation due to neuromuscular and chest wall disease.

Most patients with significant ventilatory limitation as a consequence of neuromuscular or chest wall disease progress from normal daytime arterial oxygen tension $\left(\mathrm{PaO}_{2}\right)$ and arterial carbon dioxide tension $\left(\mathrm{PaCO}_{2}\right)$ to nocturnal hypoventilation initially in rapid eye movement (REM) sleep. This then extends to non-REM sleep and, if vital capacity falls further, daytime chronic ventilatory failure ensues. ${ }^{8}$ Patients progress at different rates through this pathophysiological sequence, so that some individuals present with uncontrolled ventilatory failure and may require emergency treatment in the intensive care unit (ICU) while others decompensate at the time of chest infections. As waiting for the development of daytime ventilatory failure may expose the patient to the risk of uncontrolled decompensation and treatment too early has been shown to be ineffective, the current trial was designed to test the hypothesis that application of NIV to control nocturnal hypoventilation in the absence of diurnal ventilatory failure is a valid time to introduce NIV.

\section{METHODS}

Study subjects

Patients with congenital neuromuscular and chest wall disease were recruited from neuromuscular clinics at Royal Brompton Hospital over the period 1997-2000. Entry criteria were neuromuscular or chest wall disease established by

\footnotetext{
Abbreviations: $\mathrm{CPF}$, cough peak flow; FRC, functional residual capacity; NIV, non-invasive ventilation; $\mathrm{PaO}_{2}$, arterial oxygen tension; $\mathrm{PaCO}_{2}$, arterial carbon dioxide tension; PImax, maximum inspiratory mouth pressure; PEmax, maximum expiratory mouth pressure; SNIP, sniff inspiratory pressure; $\mathrm{TcCO}_{2}$, transcutaneous carbon dioxide tension
} 
muscle biopsy, genetic analysis, or confirmed by clinical characteristics and specialist neurological opinion. All patients with a vital capacity of less than $50 \%$ predicted or symptoms suggestive of nocturnal hypoventilation (poor sleep quality, morning headaches, waking with breathlessness) were assessed by clinical examination, measurement of daytime arterial blood gas tensions breathing air and respiratory muscle strength, and an overnight respiratory sleep study including monitoring of nocturnal arterial oxygen saturation $\left(\mathrm{SaO}_{2}\right)$ and transcutaneous carbon dioxide tension $\left(\mathrm{TCCO}_{2}\right)$. Patients with a normal daytime $\mathrm{PaCO}_{2}$ but nocturnal hypoventilation confirmed by a peak $\mathrm{TCCO}_{2}>6.5 \mathrm{kPa}$ were randomised to a control group who were not treated with NIV but underwent 6 monthly reassessment for 24 months (group 1), or to a group started immediately on nocturnal NIV (group 2). Group 2 were also followed by 6 monthly assessments for 24 months. For safety reasons a priori criteria were set so that, if patients in the control group 1 fulfilled any one of these, NIV would be started. The criteria were: development of daytime hypercapnia $\left(\mathrm{PaCO}_{2}\right.$ $>6.5 \mathrm{kPa}$ ), worsening symptoms of nocturnal hypoventilation, recurrent chest infections ( $>3$ per year), failure to thrive in children, and acute ventilatory decompensation requiring hospitalisation. The time at which patients met any of these criteria was recorded. Patients with a daytime $\mathrm{PaCO}_{2}$ $>6.5 \mathrm{kPa}$ met established criteria to begin NIV and therefore were excluded from randomisation and started on NIV (group 3).

Ovenight $\mathrm{SaO}_{2}$ was monitored using a Nellcor N200 pulse oximeter (Tyco, Gosport, UK) and $\mathrm{TCCO}_{2}$ was measured using a TINA TcCM $\mathrm{CO}_{2}$ electrode (Radiometer, Copenhagen, Denmark) heated to $42.5^{\circ} \mathrm{C}$ and placed on the forearm following careful skin preparation. The Radiometer TINA system has been previously validated in our laboratory by comparison with simultaneous arterial blood gas sampling ${ }^{9}$ and was calibrated using standard $\mathrm{CO}_{2}$ test gas mixtures before each sleep study.

Respiratory muscle tests comprised non-invasive measures. Maximum inspiratory mouth pressure strength (PImax) and maximum expiratory mouth pressure (Pemax) were measured from functional residual capacity (FRC) using a mouthpiece, sniff inspiratory pressure (SNIP) was measured from the contralateral nostril during 10 maximal sniffs from FRC, and cough peak flow (CPF) was assessed by a maximal cough effort into a mouthpiece connected to a pneumotachograph (Fleisch no 4 head). All signals were measured using a differential pressure transducer (Validyne, USA). Patients completed SF-36 health related quality of life questionnaires at baseline and at each 6 monthly assessment.

\section{Ventilatory support}

NIV was provided using pressure targeted ventilators: BiPAP ST (Respironics, Murrysville, USA), VPAP ST II, (ResMed Ltd, Oxfordshire, UK) and Breas PV401 (Vital Signs, Littlehampton, UK) in spontaneous timed mode. Ventilatory settings were established during overnight monitoring on a separate night to the diagnostic sleep study in order correct $\mathrm{SaO}_{2}$ and to normalise $\mathrm{TcCO}_{2}$. Nasal or face masks (Respironics Co, ResMed Ltd) were used according to patient comfort and compliance was monitored using hour counters in the ventilators. Patients were advised to use the ventilator at night while they were asleep, and during physiotherapy sessions to improve cough efficacy and secretion clearance when they developed a chest infection. Supplemental oxygen therapy was not used.

\section{Analysis of data}

Peak $\mathrm{TCCO}_{2}$ was used as the primary outcome measure. In order to demonstrate significant improvement in nocturnal

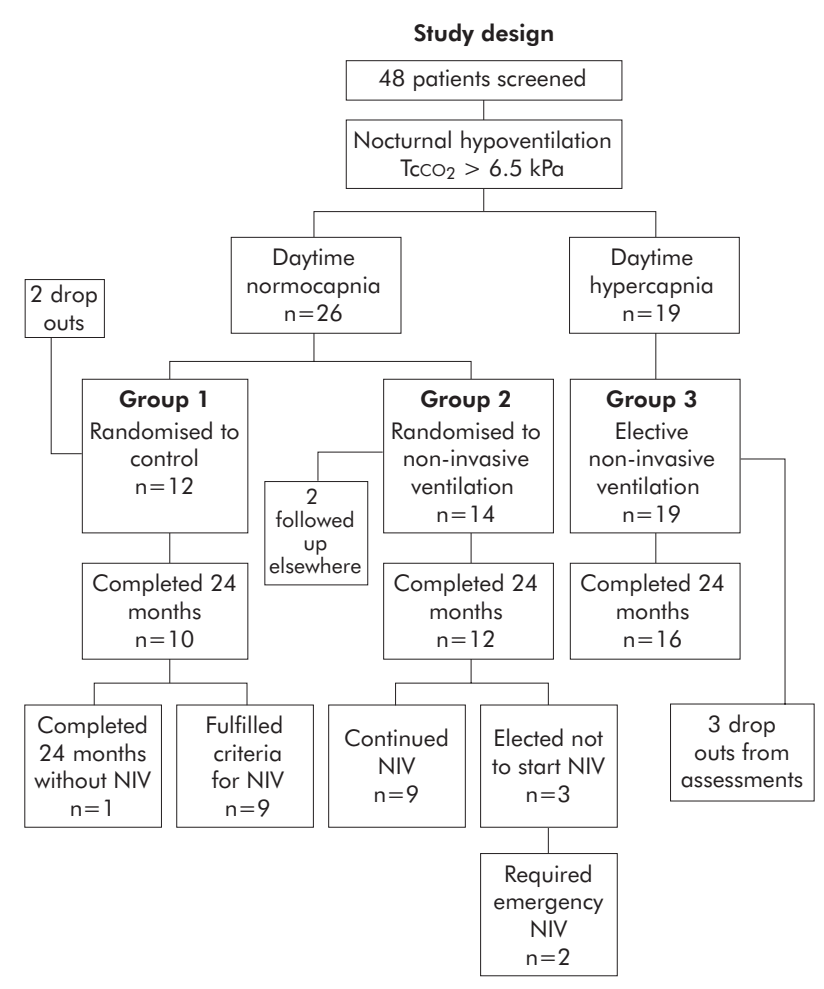

Figure 1 Study design.

hypoventilation (mean (SD) fall in peak $\mathrm{TcCO}_{2}$ by 2 (1) $\mathrm{kPa}$ ) with a power of 0.9 and $p=0.05$, a minimum of seven patients needed to be randomised to each group. We selected this value as several hundred previous sleep studies in this unit suggested that mean overnight peak $\mathrm{TCCO}_{2}$ was likely to be around $8.5 \mathrm{kPa}$. As the effect of intervention failure was not clear, a priori criteria were set which were in line with standard indications for NIV such that, if patients met these, NIV was started. Other key outcomes were therefore the number of patients who met criteria to initiate NIV in the control group and changes in pulmonary function, respiratory muscle strength, and SF-36 quality of life domains. To obtain more continuous measures of arterial blood gas control we also calculated the period of overnight monitoring during which $\mathrm{TcCO}_{2}$ exceeded $6.5 \mathrm{kPa}\left(\mathrm{TcCO}_{2}>6.5 \mathrm{kPa}\right)$ and mean overnight $\mathrm{SaO}_{2}$. A similar sample size $(\mathrm{n}=7$ in each group) was required to demonstrate a reduction in the time during which $\mathrm{TCCO}_{2}>6.5 \mathrm{kPa}$ from $70 \%$ to $30 \%$ (SD 20) with a power of 0.9 and $\mathrm{p}=0.05$.

The difference between baseline values and values at 6,12 , 18 and 24 months was compared between groups 1 and 2 at each time point using an unpaired $t$ test. Arterial blood gas and SF-36 results from group 3 (patients with daytime hypercapnia) were analysed using ANOVA to establish whether patients with more pronounced ventilatory insufficiency had a different outcome.

As $70 \%$ of group 1 patients had started NIV by 12 months, thereby reducing the chance of detecting a significant difference between groups, post hoc comparison of the change in physiological variables from baseline to values at 6 months in groups 1 and 2 was performed using an unpaired $t$ test. Analyses were carried out using SigmaStat 3.0 or SPSS 12.0 .

\section{RESULTS}

Forty eight patients were screened for entry into the study (fig 1); 45 of these had nocturnal hypoventilation and daytime $\mathrm{PaCO}_{2}$ was raised in 19. These patients (group 3) 
Table 1 Patient characteristics on entry to the trial

\begin{tabular}{|c|c|c|c|c|}
\hline Diagnosis & $\begin{array}{l}\text { Age } \\
\text { (years) }\end{array}$ & $\begin{array}{l}\text { Diurnal } \mathrm{PaO}_{2} \\
(\mathrm{kPa})\end{array}$ & $\begin{array}{l}\text { Diurnal } \mathrm{PaCO}_{2} \\
(\mathrm{kPa})\end{array}$ & $\begin{array}{l}\text { Vital capacity } \\
\text { (I) }\end{array}$ \\
\hline $\begin{array}{l}\text { Group 1: Control } \\
\text { DMD }(n=3) \\
\text { CMD }(n=2) \\
\text { BMD }(n=1) \\
\text { CMT }(n=1) \\
\text { SMA II }(n=1) \\
\text { Beal's syndrome }(n=1) \\
\text { Osteogenesis imperfecta }(n=1)\end{array}$ & $18(7-46) ; 21(11)$ & $10.27(1.4)$ & $6.03(0.39)$ & $0.81(0.61)$ \\
\hline $\begin{array}{l}\text { Group 2: Randomised to NIV } \\
\text { DMD }(n=2) \\
\text { CMD }(n=3) \\
\text { Congenital KS }(n=3) \\
\text { Poland's syndrome }(n=1)\end{array}$ & $18(11-51) ; 26(14)$ & $10.70(0.87)$ & $5.79(0.66)$ & $1.01(0.34)$ \\
\hline $\begin{array}{l}\text { Group 3: Elective NIV } \\
\text { DMD }(n=8) \\
\text { CMD }(n=3) \\
\text { LGMD }(n=1) \\
\text { Congenital KS }(n=4)\end{array}$ & $22.5(10-66) ; 28.3(15.6)$ & $10.19(2.23)$ & $7.47(1.92)$ & $0.53(0.19)$ \\
\hline
\end{tabular}

were electively started on NIV and 16 attended for regular study assessments. The remaining 26 patients with nocturnal hypoventilation but daytime normocapnia were randomised to control group 1 or to start NIV (group 2). The diagnoses, age, vital capacity, and daytime arterial blood gas tensions of these patients are shown in table 1.

Changes in nocturnal blood gas tensions from baseline values are shown in fig 2 . From an initial mean (SD) value of $9.22(1.08) \mathrm{kPa}$ in group 1 and $9.56(0.56) \mathrm{kPa}$ in group 2, peak $\mathrm{TCCO}_{2}$ did not change significantly between groups with a mean (SD) fall of $1.33(1.96) \mathrm{kPa}$ in group 1 and 2.7 (0.94) $\mathrm{kPa}$ in group $2(\mathrm{p}=0.5,95 \% \mathrm{CI}-6.04$ to 3.30$)$ by 24 months. However, the percentage of time $\mathrm{TcCO}_{2}$ exceeded
$6.5 \mathrm{kPa}$ decreased significantly by $-11.75(46.1) \%$ from a mean (SD) baseline value of $71.9(29.6) \%$ in group 1 and by $-57.7(26.2) \%$ from a baseline value of $77.2(12.2) \%$ in group 2 (mean difference $\mathrm{p}=0.049,95 \% \mathrm{CI}-91.5$ to -0.35$)$. Mean (SD) overnight $\mathrm{SaO}_{2}$ increased from 93.5 (1.9)\% to 96.55 (1.2)\% in the NIV group compared with a change from 95.2 (2.1)\% to 93.55 (1.7)\% in the control group (mean difference $\mathrm{p}=0.024,95 \%$ CI 0.69 to 7.5$)$. The mean minimum $\mathrm{SaO}_{2}$ did not differ between groups at 24 months. Post hoc analysis showed that both percentage time $\mathrm{TCCO}_{2}>6.5 \mathrm{kPa}$ and mean $\mathrm{SaO}_{2}$ improved significantly in group 2 patients compared with controls at 6 months but there was no difference in peak $\mathrm{TcCO}_{2}$ and minimum $\mathrm{SaO}_{2}$. Daytime arterial blood gas
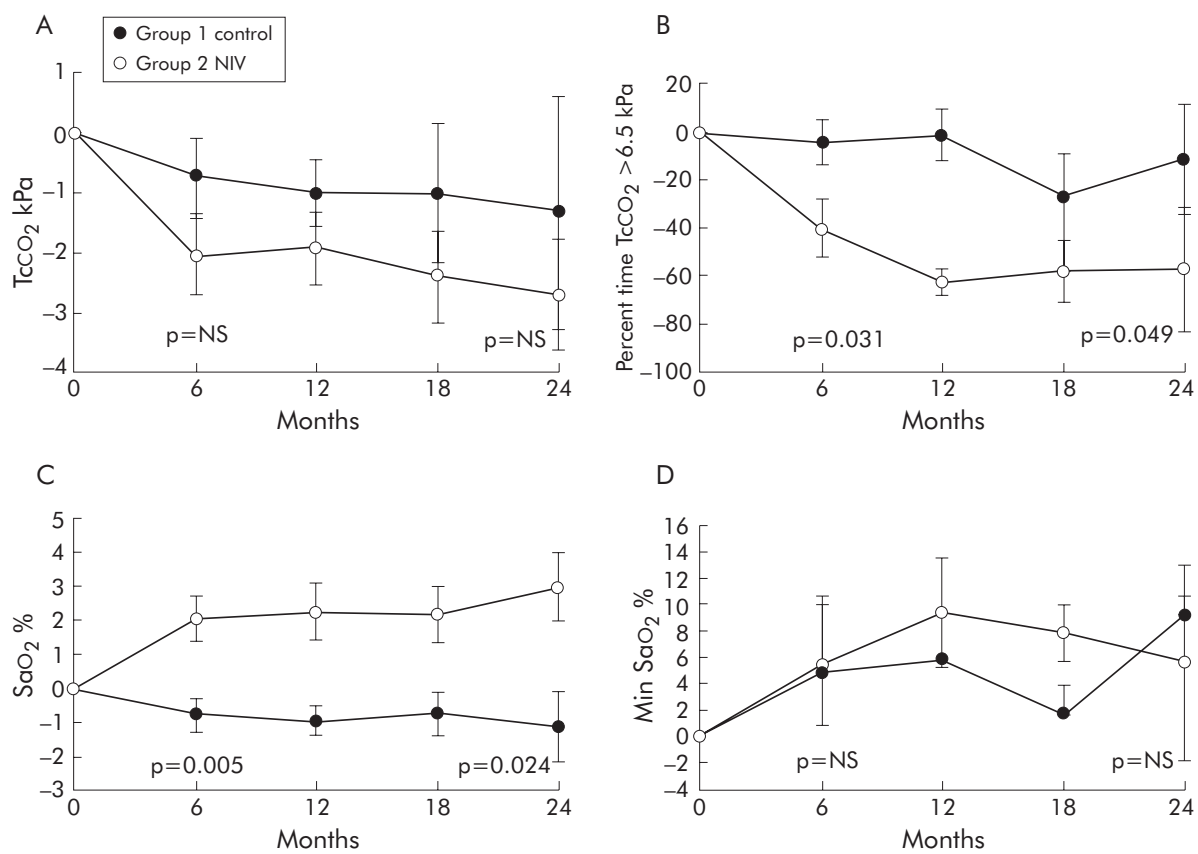

D

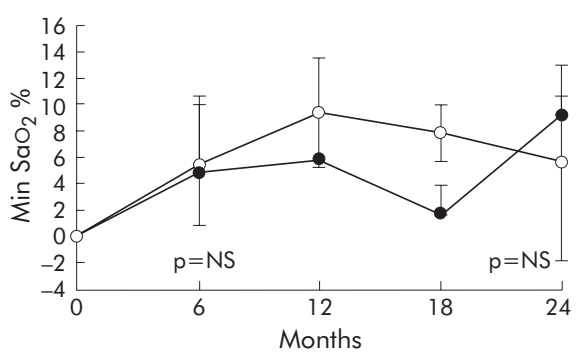

Figure 2 Difference between baseline values and those at 6, 12, 18 and 24 months in (A) peak nocturnal Tcco, (B) mean nocturnal time TccO $>6.5 \mathrm{kPa},(\mathrm{C})$ mean nocturnal $\mathrm{SaO}_{2}$, and (D) mean minimum nocturnal $\mathrm{SaO}_{2} ; \mathrm{p}$ values given at 6 and 24 months. Error bars represent $\mathrm{SE}$. 


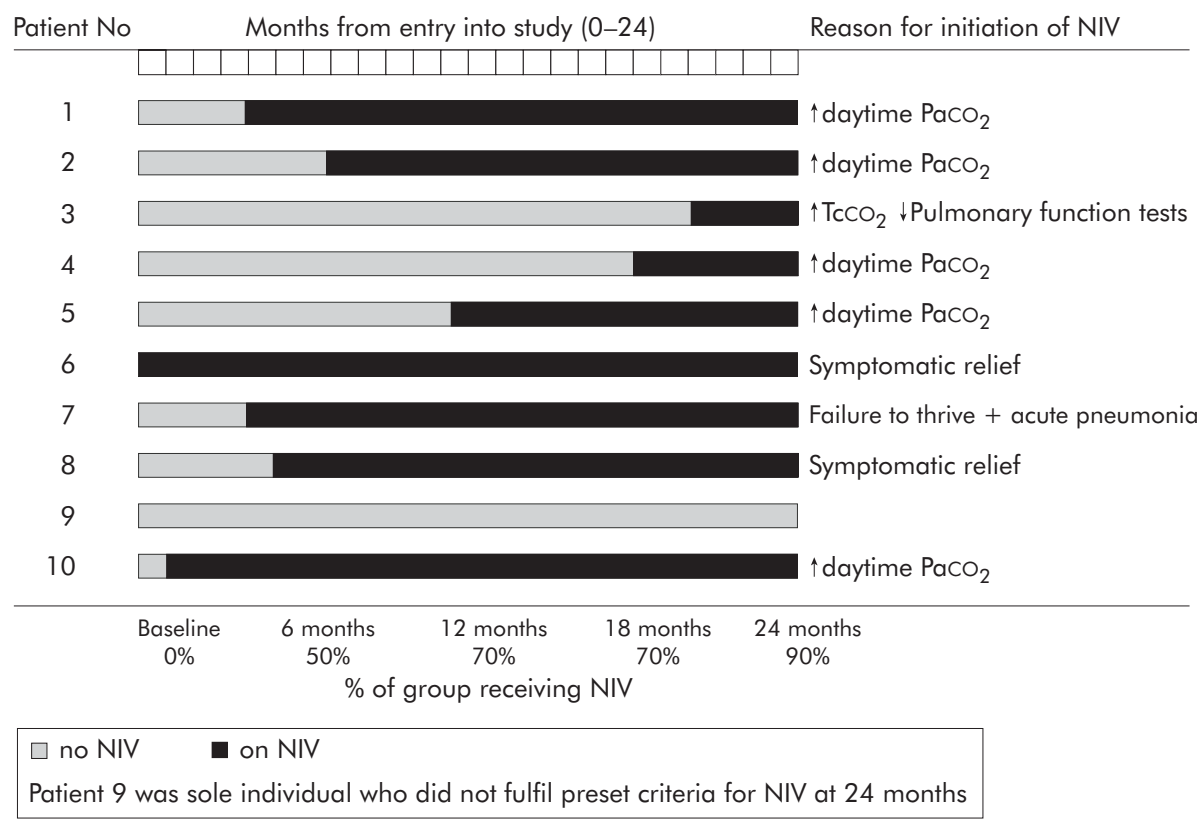

Figure 3 Reasons for group 1 (control) patients fulfilling preset criteria and time at start of NIV.

tensions did not change significantly in groups 1 and 2 but mean (SD) $\mathrm{PaCO}_{2}$ fell from 7.47 (1.9) kPa to 5.98 (0.97) $\mathrm{kPa}$ in group $3(\mathrm{p}<0.003)$.

Ten patients competed the 24 month trial period in group 1 and nine in group 2, as shown in fig 1 . In the control group, seven patients met the preset criteria for starting NIV within the first 12 months and, by 24 months, only one patient continued without NIV. The reasons for meeting the criteria and time at which the criteria were fulfilled are shown in fig 3. Seven patients developed daytime hypercapnia with a mean (SD) daytime $\mathrm{PaCO}_{2}$ increasing from $6.22(0.22) \mathrm{kPa}$ to $7.78(0.47) \mathrm{kPa}$, three experienced worsening symptoms of nocturnal hypoventilation including intractable morning headache, and one child had poor weight gain and developed pneumonia.

In group 2, two patients randomised to receive NIV decided they did not want to start this because of the inconvenience. Both were admitted within the 24 month follow up period with uncontrolled hypercapnia and were started on NIV as an emergency, one requiring ICU admission. One patient failed to attend follow up appointments regularly, one opted out of the study, and one patient moved away and received follow up elsewhere uneventfully. Nine patients in group 2 completed the 24 month follow up assessments using nocturnal NIV. Of the 19 patients in group 3 with daytime hypercapnia who fulfilled Consensus conference recommendations $^{6}$ to start NIV immediately, three discontinued the regular trial assessments and 16 completed the 24 month follow up period.

There was an initial trend to lower vital capacity and respiratory muscle strength in group 1 but this was not significant. Baseline mean (SD) values for Pimax, Pemax, SNIP, and cough peak flow were 35.4 (12.2) $\mathrm{cm} \mathrm{H}_{2} \mathrm{O}, 48.8$ (35.0) $\mathrm{cm} \mathrm{H}_{2} \mathrm{O}, 29.0(24.5) \mathrm{cm} \mathrm{H}_{2} \mathrm{O}$, and $162.8 \mathrm{l} / \mathrm{min}$, respectively, in group $\mathrm{l}$, and $55.0 \quad(27.8) \mathrm{cm} \mathrm{H} \mathrm{H}_{2} \mathrm{O}, \quad 77.7$ (22.7) $\mathrm{cm} \mathrm{H}_{2} \mathrm{O}, 52.8$ (22.4) $\mathrm{cm} \mathrm{H}_{2} \mathrm{O}$, and 206.8 (80.4) l/min in group 2 . Over the 24 month study period there was no change from baseline in vital capacity, Pimax, Pemax, SNIP, and cough peak flow in groups 1 and 2. Mean (SD) changes in SNIP and cough peak flow by the end of the study were 1.62 (6.1) $\mathrm{cm} \mathrm{H}_{2} \mathrm{O}$ and 43.3 (52.2) $\mathrm{l} / \mathrm{min}$ in group $\mathrm{l}$ and -4.1 (14.1) $\mathrm{cm} \mathrm{H}_{2} \mathrm{O}$ and -0.9 (34.4) $\mathrm{l} / \mathrm{min}$, respectively, in group 2.
In group 3 the mean (SD) vital capacity before NIV was 0.53 (0.19) $\mathrm{l}$ and $0.51(0.24) \mathrm{l}$ after 24 months, with mean (SD) Pimax, Pemax, SNIP, and cough peak flow values of 20.9 (15.7) cm $\mathrm{H}_{2} \mathrm{O}, 31.0$ (33.0) $\mathrm{cm} \mathrm{H}_{2} \mathrm{O}, 22.1$ (19.5) $\mathrm{cm} \mathrm{H}_{2} \mathrm{O}$, and $131.8 \mathrm{l} / \mathrm{min}$, respectively, at entry to the study. There was no significant change in PImax, Pemax, and SNIP at 24 months but cough peak flow fell significantly to $113.9 \mathrm{l} / \mathrm{min}$ $(\mathrm{p}=0.013,95 \% \mathrm{CI}-50.5$ to -10.7$)$.

Compared with the control patients, a gain in the SF-36 general health score was seen in group 2 patients by 18 months ( $p=0.035,95 \%$ CI 51.35 to 2.31 ) but there were no changes in other domains in groups 1 and 2. In group 3, vitality and social function scores improved $(p=0.017$ and $p=0.031$, respectively) and there was a trend to improvement in general health $(p=0.06)$ and mental health $(\mathrm{p}=0.058)$ domains during the 2 year study period.

Mean (SD) and median (range) ventilator use was 4.65 $(2.2), 4.79(1.36-7.4)$ hours per night in group 2 patients and $6.2(2.5), 6.1$ (1.4-11.1) hours in patients in group 1 (although group 1 included use in a 7 year old child with a longer bed time). There was no significant difference in compliance between groups 1 and 2 . Ventilator compliance data were available in 12 of 16 patients in group 3 who completed the follow up measurements and showed mean (SD) and median (range) values of 9.07 (2.96), 9.16 (2.0413.45) hours. Mean ventilator settings were inspiratory pressure $18 \mathrm{~cm} \mathrm{H}_{2} \mathrm{O}$ and expiratory positive pressure $3 \mathrm{~cm} \mathrm{H}_{2} \mathrm{O}$ (the PV403 ventilator we used does not provide expiratory positive pressure).

\section{DISCUSSION}

In patients with congenital myopathies and muscular dystrophies, Ragette et $a l^{8}$ have shown that a vital capacity of less than $60 \%$ predicted is associated with the development of REM related hypopnoeas and a vital capacity of less than $40 \%$ predicted and maximum inspiratory pressure of less than $30 \mathrm{~cm} \mathrm{H}_{2} \mathrm{O}$ are strongly correlated with the presence of nocturnal hypoventilation in both REM and NREM sleep. Daytime ventilatory failure is virtually inevitable once vital capacity falls below $25 \%$ predicted. While there has been no randomised controlled trial of NIV in neuromuscular patients with chronic daytime hypercapnia, Vianello et al have 
shown that the average life expectancy in men with Duchenne muscular dystrophy without ventilatory support is approximately 9-10 months. As the outcome of respiratory failure is so poor and the life prolonging effects of NIV so clearcut, ${ }^{11-13}$ it has been suggested that a randomised controlled trial in hypercapnic patients would be unethical. ${ }^{14}$ Large cohort series, some using historical comparisons, have shown that long term NIV produces a significant improvement in survival and quality of life in adults and children with stable chest wall and neuromuscular disease. ${ }^{1215}$ Survival advantage $\mathrm{e}^{1617}$ and increments in quality of life $\mathrm{e}^{18} 19$ have also been reported in patients with progressive neuromuscular disease such as amyotrophic lateral sclerosis/motor neurone disease. The British Thoracic Society guidelines on $\mathrm{NIV}^{20}$ suggest that all such patients who have an episode of ventilatory failure should be referred to a specialist centre for consideration of home ventilation, but these recommendations focus predominantly on the delivery of acute NIV rather than chronic application in the home or the prevention of ventilatory decompensation. As mentioned above, US Consensus Conference guidelines ${ }^{6}$ for patients with restrictive thoracic disorders or central hypoventilation recommend NIV in the presence of symptoms of nocturnal hypoventilation and nocturnal arterial oxygen desaturation $\left(\mathrm{SaO}_{2}<88 \%\right.$ for more than 5 minutes or for greater than $10 \%$ of total monitoring time). However, as pointed out by Mehta and Hill, ${ }^{21}$ these nocturnal desaturation criteria were not based on evidence but were derived from Medicare guidelines for oxygen therapy in chronic obstructive pulmonary disease. Oxygen therapy in neuromuscular patients is usually contraindicated in view of the risk of provoking uncontrolled hypercapnia. As a result of scant evidence to support the use of NIV in nocturnal hypoventilation, this indication was not supported in a recent Cochrane review. ${ }^{7}$

In an uncontrolled study, Mellies et $a^{3}$ have applied long term NIV in children and adolescents with a variety of congenital neuromuscular disorders (intermediate spinal muscular atrophy, congenital muscular dystophy and myopathies) who presented with either nocturnal hypoventilation or diurnal ventilatory failure. Although improvement in daytime arterial blood gas tensions was greater in the group with daytime ventilatory failure, all tolerated NIV well and there were similar improvements in sleep quality and symptoms. In the light of these findings and other uncontrolled studies, we felt the current trial was justified to clarify indications for NIV before the development of daytime ventilatory failure.

\section{Limitations of trial}

The trial can be criticised for including a heterogeneous group of patients with a variety of disorders all of which might have a differing natural history. However, these conditions are relatively uncommon and have a variable phenotype, such that ventilatory limitation is caused not only by the extent of respiratory muscle weakness but also by other factors such as the presence of a thoracic scoliosis and the degree of expiratory muscle weakness which may predispose the individual to chest infections. It would therefore be extremely difficult to carry out this study with completely matched groups. In addition, in clinical practice patients are referred with a variety of conditions and decisions need to be made on an individual basis. All patients had a primary congenital neuromuscular disorder except several who had a severe congenital scoliosis, in some cases associated with inherited syndromes (Poland's syndrome, Beal's syndrome). These scoliosis patients also had confirmed respiratory muscle insufficiency, but this was less marked than in those with neuromuscular disease and may be attributable to the respiratory muscles working at a mechanical disadvantage.
The sole individual in the control group who did not fulfil criteria for NIV within 24 months had a congenital scoliosis secondary to Beal's syndrome, perhaps indicating that, in those with predominantly chest wall disease rather than progressive respiratory muscle weakness, ventilatory insufficiency may evolve more slowly. In addition, nocturnal hypoventilation was first observed in this patient during an adolescent growth spurt and it should be noted that, in some children, a rapid skeletal growth spurt can sometimes outstrip the capacity of respiratory muscles to maintain ventilation overnight until a catch-up increase in respiratory muscle strength occurs.

The numbers included in this study are small, increasing the risk of unrepresentative findings. However, the results show such a high level of failure of conservative management in the control group that we felt it unreasonable to continue the study. On an intention to treat basis, nine of 10 patients fulfilled the preset criteria for NIV within 24 months of an initial sleep study demonstrating nocturnal hypoventilation and, indeed, 70\% had failed conservative management within a year. In addition, two patients randomised to NIV elected not to receive this and both developed ventilatory decompensation within 12 months. Hence, on a treatment efficacy basis, 11 of 12 patients with nocturnal hypoventilation fulfilled criteria for NIV. Furthermore, by the end of the study, almost all patients in the control group were actually receiving NIV having met the preset criteria, thereby reducing the ability to differentiate between the groups on treatment effect alone.

The diagnosis of nocturnal hypoventilation rested on $\mathrm{TcCO}_{2}$ values. We have found these measurements accurate with good agreement between arterial and transcutaneous measurements, as have other groups. ${ }^{22}{ }^{23}$ Normal values for overnight $\mathrm{CO}_{2}$ are limited and many studies use the end tidal $\mathrm{CO}_{2}$ measurement which is not directly comparable. It is also more difficult to apply because of displacement of the probe and the need to obtain follow up measurements while the individual is receiving mask ventilation. It has been suggested that nocturnal hypoventilation is defined by a rise in overnight $\mathrm{PaCO}_{2}$ of $>1.33 \mathrm{kPa}(10 \mathrm{~mm} \mathrm{Hg})$ or a $\mathrm{PaCO}_{2}$ of $>6.7 \mathrm{kPa}$ for $50 \%$ or more of sleep time, ${ }^{24}$ but this is based on a small study of normal children in which hypopnoeas ${ }^{25}$ were not included. We selected a cut-off value for peak $\mathrm{TCCO}_{2}$ $>6.5 \mathrm{kPa}$ as this is easy to define, and because it proved predictive of worsening nocturnal hypoventilation and a high risk of decompensation during a chest infection in a series of over 400 neuromuscular patents referred to the Royal Brompton Hospital. In practice, patients in groups 1 and 2 had a $\mathrm{TCCO}_{2}$ value of $>6.5 \mathrm{kPa}$ for more than $70 \%$ of the total study period at baseline investigation. All studies were visually inspected (AKS) to ensure that the peak $\mathrm{TCCO}_{2}$ value did not represent an artifact caused by rapid movement of the electrode. Several different types of pressure targeted ventilators were used but, as the aim was to control nocturnal hyopoventilation, we do not believe that this affected the results and it is representative of what occurs in clinical practice.

Finally, the addition of expiratory muscle support with cough in-exsufflator assistance has been recommended in neuromuscular patients. ${ }^{26}{ }^{27}$ In this study, cough in-exsufflators were not prescribed as they were not widely available or approved for use in Europe at the start of the study, but patients were shown how to use the non-invasive ventilator as a physiotherapy adjunct to assist sputum clearance at the time of chest infections. ${ }^{28}$ It is notable that cough peak flow decreased significantly in group 3 patients whereas measures of inspiratory muscle strength remained stable in all groups, suggesting that a combination of inspiratory and expiratory muscle assistance may be helpful in those with advanced disease. 
In conclusion, the findings of this study suggest that, regardless of the underlying condition, patients with nocturnal hypoventilation secondary to neuromuscular disease are at risk of daytime ventilatory failure and this is likely to ensue within the following 12-24 months. This information can be discussed with individuals and their families, allowing them time to consider the practical implications of ventilatory support and participate in decision making so that the initiation of NIV can be planned around school, college, work and family commitments, and medical teams can plan work load and make provision for ventilatory supplies. Although improvements in nocturnal and daytime arterial blood gas tensions and quality of life are not as great when NIV is applied to treat nocturnal hypoventilation rather than diurnal ventilatory failure, considered intervention with NIV to control nocturnal hypoventilation may reduce the frequency of acute hypercapnic crises provoking general ward and ICU admissions. As fewer patients with pure chest wall disorders were included, the results may be less applicable to this group than to patients with neuromuscular disease.

\section{ACKNOWLEDGEMENTS}

The authors are grateful to M Morrell and G Meadows for technical support, and M Khan, Research Services, Royal Brompton Hospital for statistical advice.

\section{Authors' affiliations}

S Ward, M Chatwin, S Heather, A K Simonds, Clinical and Academic Department of Sleep and Breathing, Royal Brompton \& Harefield NHS Trust, London SW3 6NP, UK

Competing interest: AKS is in receipt of research grants from Breas Medical and ResMed UK.

The study was approved by the ethics committee of the Royal Brompton \& Harefield NHS Trust and informed consent was obtained from all participants or their parents.

\section{REFERENCES}

1 Leger P, Bedicam JM, Cornette A, et al. Nasal intermittent positive pressure ventilation. Long term follow-up in patients with severe chronic respiratory insufficiency. Chest 1994;105:100-5.

2 Simonds AK, Elliott MW. Outcome of domiciliary nasal intermittent positive pressure ventilation in restrictive and obstructive disorders. Thorax 1995;50:604-9.

3 Mellies U, Ragette R, Schwake C, et al. Long-term noninvasive ventilation in children and adolescents with neuromuscular disorders. Eur Respir $J$ 2003;22:631-6.

4 Raphael J-C, Chevret S, Chastang C, et al. Randomised trial of preventive nasal ventilation in Duchenne muscular dystrophy. Lancet 1994;343:1600-4.

5 American Thoracic Society Consensus statement. Respiratory care of the patient with Duchenne muscular dystrophy. Am J Respir Crit Care Med 2004; 170:456-65.
6 Consensus Conference. Clinical Indications for noninvasive positive pressure ventilation in chronic respiratory failure due to restrictive lung disease, COPD, and nocturnal hypoventilation: a consensus conference report. Chest 1999;116:521-34.

7 Annane D, Chevrolet JC, Chevret S, et al. Nocturnal mechanical ventilation for chronic hypoventilation in patients with neuromuscular and chest wall disorders. Cochrane Database of Systematic Reviews 2000;(2):CD001941.

8 Ragette R, Mellies U, Schwake C, et al. Patterns and predictors of sleep disordered breathing in primary myopathies. Thorax 2002;57:724-8.

9 Simonds AK. Selection of patients for home ventilation. In: Simonds AK, ed. Non-invasive respiratory support. London: Arnold, 2001:119-32.

10 Vianello A, Bevilacqua $M$, Salvador V, et al. Long-term nasal intermittent positive pressure ventilation in advanced Duchenne's muscular dystrophy. Chest 1994;105:445-8.

11 Jeppesen J, Green A, Steffensen BF, et al. The Duchenne muscular dystrophy population in Denmark, 1977-2001: prevalence, incidence and survival in relation to the introduction of ventilator use. Neuromusc Disord 2003;13:804-12

12 Eagle M, Baudouin S, Chandler C, et al. Survival in Duchenne muscular dystrophy: improvements in life expectancy since 1967 and the impact of home nocturnal ventilation. Neuromusc Disord 2002;12:926-9.

13 Simonds AK, Muntoni F, Heather S, et al. Impact of nasal ventilation on survival in hypercapnic Duchenne muscular dystrophy. Thorax 1998; 53:949-52.

14 Hill NS. Noninvasive positive pressure ventilation in neuromuscular disease. Enough is enough! Chest 1994;105:337-8.

15 Simonds AK, Ward S, Heather S, et al. Outcome of paediatric domiciliary mask ventilation in neuromuscular and skeletal disease. Eur Respir J 2000;16:476-81

16 Pinto AC, Evangelista T, Carvalho M, et al. Respiratory assistance with a noninvasive ventilator (BiPAP) in MND/ALS patients: survival rates in a controlled trial. J Neurol Sci 1995; 129(Suppl): 19-26.

17 Kleopa KA, Sherman M, Bettie N, et al. BiPAP improves survival and rate of pulmonary function decline in patients with ALS. J Neurol Sci 1999;164:82-8.

18 Bourke SC, Shaw PJ, Gibson GJ. Respiratory function vs sleep disordered as predictors of QOL in ALS. Neurology 2001;57:2040-4.

19 Lyall RA, Donaldson N, Fleming T, et al. A prospective study of quality of life in ALS patients treated with non-invasive ventilation. Neurology 2001;57:153-6.

20 British Thoracic Society Standards of Care Committee. Non-invasive ventilation in acute respiratory failure. Thorax 2002;57:192-211.

21 Mehta S, Hill NS. State of the art. Noninvasive ventilation. Am J Respir Crit Care Med 2001; 163:540-77.

22 Milross MA, Piper AJ, Norman M, et al. Low-flow oxygen and bilevel ventilatory support. Effects on ventilation during sleep in cystic fibrosis. Am J Respir Crit Care Med 2001;163:129-34.

23 Janssens JP, Perrin E, Bennani $L$, et al. Is continuous transcutaneous monitoring of $\mathrm{PCO}_{2}\left(\mathrm{TcPCO}_{2}\right)$ over $8 \mathrm{~h}$ reliable in adults? Respir Med 2001;95:331-5.

24 Wallgren-Pettersen C, Bushby K, Mellies U, et al. Ventilatory support in congenital neuromuscular disorders: congenital myopathies, congenital muscular dystrophies, congenital myotonic dystrophy amd SMA II. Neuromusc Disord 2004;14:56-69.

25 Marcus CL, Omlin KJ, Basinski DJ, et al. Normal polysomnographic values for children and adolescents. Am Rev Respir Dis 1992;146:1235-9.

26 Bach JR. Mechanical insufflation-exsufflation. Comparison of peak expiratory flows with manually assisted and unassisted coughing techniques. Chest 1993; 104:1553-62.

27 Bach JR, Ishikawa Y, Kim H. Prevention of pulmonary morbidity for patients with Duchenne muscular dystrophy. Chest 1998;112:1024-8.

28 Bott J, Agent P, Callaghan S. Physiotherapy and nursing during non-invasive ventilation. In: Simonds AK, ed. Non-invasive respiratory support. London: Arnold, 2001:231-54. 